\title{
Del Manifiesto a la Internet: relaciones entre texto escrito, literatura y creación performática en Latinoamérica*
}

Fecha de recepción: 20 de Agosto de 2018

Fecha de aprobación: 18 de diciembre de 2018

\section{Resumen}

Este trabajo aborda el diálogo entre texto escrito y creación artística en performance, básicamente a partir de la praxis de esta última, centrándonos en las formas por medio de las cuales la performance se nutre del texto escrito. Se analiza al texto escrito como elemento en la construcción de las piezas; como registro; como paratexto; a la escritura sobre performance; a las peculiaridades de la relación entre texto escrito literario y performance, y las posibilidades que se presentan bajo el alero de las nuevas tecnologías. Se utiliza una metodología exploratoria, que además considera las experiencias del autor en el área. Todo desde una perspectiva latinoamericana ${ }^{1}$ a partir de la cual se intenta contribuir a la construcción de un cuerpo teórico desde y hacia nuestros territorios.

Palabras clave: performance, performance y texto escrito, performance y texto escrito literario, literatura expandida, registro, performance latinoamericano.

$1 \quad$ En este aspecto se sigue a Cullen (2011), que vislumbra a Latinoamérica no como una unidad territorial perfectamente delineada, sino que la ve como una identidad, o sea un ente cultural identificable, aspecto en el que no se profundizará más allá por los objetivos de este trabajo, pero sobre el cuál se puede consultar mi tesis de maestría (2014, pp. xxxiii-xxxvi). Por ende, se incluye en dicho marco a artistas como Montañez Ortiz, quien mantuvo sus prácticas dentro de ese marco cultural.

Citar: Arenas, R (enero-junio de 2019). Del Manifiesto a la Internet: relaciones entre texto escrito, literatura y creación performática en Latinoamérica. La Palabra, (34), 85-99. 의 https://doi.org/10.19053/01218530.n34.2019.9530

\section{Rodrigo Arenas}

Investigador asociado a Translab. M.A. en Literatura. Artista visual y escritor.Translab

r_arenas@yahoo.com.

* Artículo de reflexión en el marco de una investigación continua, sobre las relaciones entre texto, escritura y performance en América Latina. 


\section{la palabra}

\section{From the Manifesto to the Internet: Relations between Written Text, Literature, Performance Art in Latin America}

\section{Abstract}

This work addresses the dialogue between written text and performance art, based on the praxis of the latter, focusing on the ways in which performance art is nourished by the written text. The written text is analyzed as an element in the construction of performance pieces; as a record; as a paratext; as a mean to discuss performance art; from the peculiarities of the relationship between literary and performance text, and the possibilities that emerge under the new technologies. An exploratory methodology is used, taking into account the author's experiences in the area, and assuming a Latin American perspective, by which we try to contribute to the construction of a theoretical body from and towards our territories.

Keywords: performance art, performance art and written text, performance art and literary written text, register, Latin American performance art.

\section{A modo de introducción}

En el contexto latinoamericano, se ha estudiado previamente tanto el texto literario, la lectura y el proceso escritural desde el punto de vista de los Performance Studies, o sea considerando a las performances como actos vitales de transferencia que transmiten saber social, memoria y un sentido de identidad a través de acciones reiteradas, constituyendo así un lente metodológico que posibilita a los investigadores analizar diversos acontecimientos (Taylor,
2015)2. También, existen trabajos que tratan sobre las formas en las que la literatura recoge elementos desde otros medios o artes $^{3}$, como en el caso de la escritura performativa ${ }^{4}$. Sin embargo, el asunto de cómo la performance creativa ha recurrido al texto escrito y a la literatura para alimentar sus procesos, es un asunto poco discutido, particularmente en Latinoamérica. Ante este diagnóstico, el presente trabajo pretende indagar sobre el diálogo entre texto es- crito y performance, no solo potenciando los procesos de creación, difusión, sostenibilidad, teoría y pedagogía del performance, sino que también intentando dilucidar cómo el texto escrito y la literatura han operado sobre la creación performática $^{5}$ latinoamericana, y dando algunas luces adicionales sobre cómo los procesos de creación literaria podrían alimentarse de la performance. Estas inquietudes se justifican por el escenario actual de la creación en

\footnotetext{
2 Sobre la lectura y escritura, se puede consultar Martos Núñez y Vivas (2010). Sobre el texto literario y lo performático, un ejemplo es Ríos (2018).

3 Por ejemplo, Rodríguez Ruíz (2018).

$4 \quad$ Véase Brijaldo Olarte (2014).

5 Tal como lo establece Ríos (2018), performático es el adjetivo que se refiere a la performance (acción o producto cultural) y no performativo, pues este remite "a los actos de habla performativos desarrollados por el filósofo del lenguaje J.L.Austin", y por lo tanto opera al nivel del lenguaje (p. 108).
} 
performance en Latinoamérica, la cual se vislumbra aún precarizada, pese a que ha sido continua y relevante para la actividad cultural y social en general; por el desafío de reinventar el texto literario en un contexto en el que el libro ha dejado de ser el principal soporte de contenidos (Rodríguez Ruíz, 2018), y por el devenir entre la creación literaria y la creación performática del autor del artículo, siendo dicho devenir algo que se repite entre varios creadores latinoamericanos (Arenas-Carter, 2018a).

Para ello, entenderemos como texto escrito a "una unidad sintáctica/semántica que tiene un carácter comunicativo, pragmático y estructurado" (Arroyave y Barreto, 2005, p. 25) representada por medio de la escritura (Halliday, 1994). Particularmente, un texto literario escrito es aquel de carácter creativo que cumple una función estética dentro de los límites de un determinado sistema de cultura (Reisz de Rivarola, 1981), como la novela y el cuento, entre otros. Por otro lado, consideraremos a la performance como un formato de creación de artes visuales (Performance Art), en el cual el cuerpo (humano o animal) presente es el principal vehículo de representación. Al respecto, la intencionalidad es un elemento central a la hora de definirlas: si el creador nombra cierta acción que involucra la presencia del cuerpo como tal, y el sistema del arte no lo invalida teóricamente, la consideraremos como una pieza de performance.

Ahora bien, cuando hablamos de la performance como formato de creación, estaremos refiriendo a un concepto amplio. En ella no solo incluiremos lo que yo denomino su forma primigenia, esto es, la acción en vivo ${ }^{6}$, sino que también podremos involucrar otras morfologías como la vídeo-performance, la foto-performance, e incluso formas más recientes y que son producto de las nuevas tecnologías, como lo que he denominado como app-performance (Arenas-Carter, 2018b). Todo esto considerando que dichos formatos enriquecen por sí mismos el lenguaje de la performance sin perder la participación del cuerpo como vehículo esencial, lo que justifica el segundo término en cada nomenclatura, o sea ellos nos interesan en su rol de mediadores (ampliar/potenciar los alcances del lenguaje performático). También, se dejan de lado prácticas literarias que pueden considerarse como tributarias de la performance y que más bien pertenecen a la literatura en el campo expandido (Borrero, 2012), como el poetry slam, el personaje literario como performer y las lecturas de poesía, a menos que sean utilizadas como elementos dentro del marco de una performance.

Para proceder, se utilizará una metodología exploratoria con el fin de propiciar un primer acercamiento al tema, centrándose en la revisión bibliográfica, pero incorporando como metodología el punto de vista planteado por Borrero respecto a las oportunidades que se abren al realizar diálogos en los que el analista participe de la función creadora, propiciando procesos de circulación entre creadores y académicos (2012). Considerando que he producido piezas de performance continuamente desde 2010, y que además me he dedicado a investigar la performance y su teoría, publicando diversos artículos y ensayos; para fines del presente estudio, me posicionaré fluidamente entre ambos puntos. Además, es en el devenir entre ambos roles en donde se originan las preocupaciones que motivan el presente artículo, pues en el caso de algunas piezas que he realizado ${ }^{7}$, la literatura ha sido un motor, un referente y/o una elemento de y en la construcción de la ellas, situación que también he observado en la práctica de muchos otros artistas. Por otro lado, académicamente provengo del área literaria, por lo que

Entendiendo esto en su sentido clásico, o sea como una pieza de performance en la que se dan "la co-presencia física del performer y la audiencia; simultaneidad temporal de la producción y la recepción; experiencia en el momento" (Auslander, 1999, p. 61).

7 Por ejemplo, la pieza Olvido (Arenas-Carter, 2017). 
también mi lugar fluye entre la teoría de la performance creativa y la literatura. Consecuentemente, utilizaré como fuentes de datos mis experiencias como creador e investigador en los mencionados ámbitos.

\section{EI texto escrito como elemen- to de la pieza}

La relación entre texto escrito y performance está lejos de ser armónica y apacible. Para empezar, se ha planteado que la performance "es el opuesto al teatro" (Wilson, 1997, p. 2). Esta es una vieja discusión que fue previamente afrontada con base en una caracterización comparativa entre performance $\mathrm{y}$ teatro que nos permita diferenciar entre una y otra (Arenas-Carter, 2018a). En dicho contraste, es importante destacar el énfasis en el texto escrito que subyace a las artes teatrales (dramaturgia) versus el énfasis en la acción en las artes performáticas. Sin embargo, esto no significa que performance y texto escrito tengan una relación de oposición.

Efectivamente, la performance en Latinoamérica surge estrechamente ligada al texto escrito, en un marco cultural en el cual este último ha ocupado y ocupa un lugar privilegiado, tal como lo destaca Rama (1998). De hecho, citando a Cullen (2011), la historia de la performance latinoamericana se inicia con las acciones destructivistas de
Montañez Ortiz en Nueva York durante 1959, las que, tal como el nombre lo indica, consistían en destruir objetos como pianos o camas en el marco de una acción de arte. Estas piezas fueron sustentadas por el Manifiesto Destructivista del mismo autor, cuya escritura se inicia en 1957 y finaliza en 1962. Montañez Ortiz continuará escribiendo sobre performance y arte en general. Ahora bien, y casi en paralelo, el artista argentino Alberto Greco inicia sus acciones en la vía pública en 1960 (Cullen, 2011), la primera de las cuales consiste en "una pegatina de carteles de estilo publicitario con la inscripción: Alberto Greco ¡Qué Grande Sos!, y Alberto Greco, el Pintor Informalista más Importante de América" (Alberto Greco ;Que grande sos!). Anteriormente, Greco había publicado relatos y poemas en diversas revistas entre 1949 y 1950, y su poemario Fiesta fue editado ese mismo año (Alberto Greco Biografía). Greco insistió con el texto literario con su novela Besos Brujos (1965), que incorpora no solo collages y dibujos, sino que también poemas (Escritos: Besos Brujos), y aportó con su Manifiesto Dito del Arte Vivo en 1962, el cual utilizó en una performance, que consistió en pegarlo por las calles de Génova (Manifiesto Dito del Arte Vivo). Dicho texto servirá como bitácora de creación no solo para Greco, sino también para las futuras generaciones de performers:
El arte vivo es la aventura de lo real. El artista enseñará a ver no con el cuadro sino con el dedo. Enseñará a ver nuevamente aquello que sucede en la calle. El arte vivo busca el objeto pero al objeto encontrado lo deja en su lugar, no lo transforma, no lo mejora, no lo lleva a la galería de arte. El arte vivo es contemplación y comunicación directa. Quiere terminar con la premeditación que significa galería y muestra. Debemos meternos en contacto directo con los elementos vivos de nuestra realidad. Movimiento, tiempo, gente, conservaciones, olores, rumores, lugares y situaciones. ARTE VIVO, movimiento DITO. Alberto Greco 24 de julio de 1962- hora 11,30. (Manifiesto Dito del Arte Vivo, s.f., párr. 2).

Por su parte, el colectivo venezolano interdisciplinario $\mathrm{El} \mathrm{Te}$ cho de la Ballena (1961-1969), considerado como el primer proyecto grupal latinoamericano que trabajó la performance (Cullen, 2011), concibió sus acciones a partir de los textos que publicaron en la revista literaria Sardio en 1961, incluyendo un pre-manifiesto (Carrillo, 2007). Además, contaron con un brazo editorial que editó no solo una publicación periódica, sino que además diversos textos literarios como el poemario de uno de sus miembros, Caupolicán Ovalles y su ¿Duerme Usted Señor Presidente?, hito que es incluido, junto a varias accio- 
nes de este grupo, en la línea de tiempo de la performance de Cullen (2011), y que por su parte es un texto de relevancia literaria por sí mismo. De hecho, el trabajo de este colectivo no solo es fundamental para el mundo de la performance, sino que también para el mundo literario y poético de Venezuela. En sus exposiciones, textos eran expuestos en las paredes junto a pinturas y otro tipo de obras plásticas, mientras que en los espacios públicos el colectivo realizaba performances de protesta (Nesseldore, 2014), sentando un precedente para los creadores que en el futuro se dedicarían a trabajar en dichos espacios.

Por su parte, en 1963 el actor y arquitecto Juan José Gurrola, junto a los escritores Juan Vicente Melo, Juan García Ponce y Carlos Monsiváis, se unen en el proyecto Jazz Palabra $(\mathrm{Cu}-$ 1len, 2011), una de las primeras piezas de performance en México que incluía, entre diversas acciones, la lectura a viva voz de poemas (Pereztejada, 2014). Mientras, Alejandro Jodorowsky junto a Manuel Felguérez (1961-1963) realizaron en el mismo país, acciones que incluían la lectura de poemas y otros textos literarios, que, a su vez, podían servir de eje a la acción misma (García, 2007). Estos son solo algunos ejemplos de las primeras performances o proto-performances en las que participaba el texto escri- to. Adicionalmente, no debemos olvidar que la performance nace al alero del espíritu de las vanguardias, esto es, de la relevancia de la relación entre arte y vida (Arenas-Carter, 2018a), espíritu del cuál la literatura, y en particular la poesía, ya habían tributado.

El uso del texto escrito como elemento, recurso o estrategia de la performance se extiende hasta hoy. En el recorrido encontramos ejemplos como No Queremos Intoxicarnos (1988) del colectivo cubano Arte $\mathrm{Ca}$ lle (Leanfilms, 2009), una de las tantas piezas que rescata el recurso del cartel desde la protesta callejera. También, el texto puede desplegarse sobre espacios diferentes al papel. Por ejemplo, en la pieza Perra de Regina José Galindo (2005), la artista, utilizando un cuchi1lo, escribe dicha palabra sobre su muslo derecho (Ramírez, 2018); o el uso de la sangre como tinta, como en el caso de Ana Mendieta y su Signos de Sangre (López-Cabrales, 2006); o escribir con un lápiz labial sobre el vidrio, como María Teresa Hincapié y su pieza Vitrina (1991) (Rodríguez, 2009). De esta forma, queda claro que el texto escrito ha sido un recurso continuo en la historia de la actividad performática latinoamericana.

Ahora bien, si se analizan algunas de las primeras piezas de la historia de la performance la- tinoamericana (Cullen, 2011), nos percataremos que el texto escrito cumple dos funciones, además de la ya analizada. Primero, como forma de concebir el o los proyectos creativos (manifiesto o statement), mucho antes que este tipo de texto escrito se transformara casi en una obligación para los artistas plásticos. Segundo, como registro. Al respecto, entenderemos como registro a la documentación de la pieza de performance, ya sea por medios audiovisuales, escritos, digitales o una mezcla de ellos, y que el medio artístico asume como necesaria para demostrar que la pieza en cuestión efectivamente fue realizada. Ambos aspectos los revisaremos a continuación.

\section{El texto escrito como registro}

Auslander (2006) plantea que existen dos tipos de registro en la performance. El primero lo denomina como documental, y es aquel en el cual el (los) medio(s) utilizado(s) para registrar la pieza se limita(n) solo a documentarla y a servir de evidencia de la realización de dicha pieza, asumiéndose que la performance precede al registro y es independiente de él. Por ejemplo, en el caso de la mencionada Alberto Greco iQue grande sos!, las fotografías disponibles desempeñan el rol de registro documental. Lo mismo sucede con el escrito de la crítica de arte Raquel Tibol sobre la acción de Jodorowsky y Fel- 
guérez de 1963 (García, 2007): dichas acciones fueron independientemente del registro y este se limita a validar su existencia. La segunda nomenclatura que utiliza Auslander es la de registro teatral ${ }^{8}$, que no solo cumple las funciones anteriores, sino que además sirve de hábitat para la pieza y, por lo tanto en este caso la performance no es independiente del registro, sino que es montada con el explícito fin de ser fotografiada o filmada, $\mathrm{y}$ no tiene una existencia significativa previa como evento autónomo presentado ante una audiencia, por lo que el registro es el espacio en el que la pieza ocurre (2006). Volveremos más adelante sobre este tema.

Con respeto al rol del texto como registro documental de piezas de performance, voy a asumir que este es efectivo, al contrario de la postura de Auslander (2006), que plantea que lo escrito no puede funcionar como registro de lo performático, pues las descripciones escritas de las performances no nos permiten acceder a piezas en las que lo visual y lo auditivo desempeñan un rol fundamental. En esta discusión nos apoyaremos en Jones (1997), quien plantea que ni el texto escrito como registro ni el atestiguar directamente una performance permiten establecer una relación más o menos privilegiada con la pieza, ya que es imposible la existencia de una relación no mediada con cualquier producto cultural. O sea, estar presente en vivo durante una pieza de performance no garantiza que el espectador pueda conocer mejor dicha pieza, que en el caso de solo acceder al registro audiovisual de ella, pues en el primer caso se utiliza la mediación de la memoria (Jones, 1997) para activar el proceso cognitivo. Es más, también debemos considerar hasta qué grado el espectador cuenta con las herramientas cognitivas necesarias para acceder a una pieza: es diferente presenciar directamente una pieza de performance sin manejar ningún tipo de información sobre ella o sobre el arte de la performance, que aproximarse por medio de un texto escrito a una pieza contando con un acopio de habilidades e información relevante. Todo esto, asumiendo que hay un pacto de credibilidad entre dicho documento escrito y quien lo lee, algo similar a lo que puede darse al leer una biografía.

Yendo hacia el aspecto histórico, el registro documental textual desempeñó un rol fundamental durante los primeros años de producción latinoamericana en performance (1957 1970), por una parte debido a la inconsciencia respecto a la im- portancia del registro; por otra, debido a la falta de recursos que impedían el acceso a medios audiovisuales, además de la ya nombrada gran influencia de lo textual en ese período. Al respecto, si bien se suele pensar que el video y la fotografía son los principales medios de registro documental en la actualidad, en especial dado el actual abaratamiento y democratización de estas tecnologías, el rol del texto escrito sigue siendo fundamental, principalmente en dos aspectos.

Primero, el rol del texto escrito como registro documental se encuentra absolutamente vigente, pues lo escrito aún asume un rol fundamental en nuestra sociedad, y por lo tanto si una pieza de performance recure a estrategias tomadas de la vida social, probablemente tenga que relacionarse con el texto escrito. Por ejemplo, veamos el caso de la pieza de Marilyn Boror Edicto: Cambio de Nombre (2018). En ella, la artista cambia legalmente su nombre completo, y por ende su tarjeta de presentación (Marilyn Boror-art, 2018), que es un documento escrito que funciona como registro documental, atestiguando el nuevo nombre legal de la artista, su profesión, teléfono, mail y página de Instagram, sirviendo así como presentación en sociedad de la nueva identidad. Otro caso

$8 \quad$ El autor no explica el porqué de la elección de dicho adjetivo. Sin embargo, podemos pensar que teatral refiere a la idea del teatro como el lugar físico (hábitat), que acoge diversas manifestaciones artísticas, como obras de teatro, ballet, recitales de música, entre otros. 
en el que se ilustra esto es una de mis piezas, Terapia (2018) (2018c), en la cual el diario de vida, esta vez devenido en blog, desempeña el papel de un registro que, de hecho, está a medio camino entre el teatral y el documental, porque si bien sirve para atestiguar la realización de la pieza, también es su hábitat, ya que la autoterapia que sigue obligaba a la escritura de dicho diario como método terapéutico.

Segundo, hay textos escritos que pueden funcionar como complementos de los registros documentales de carácter audiovisual y digital (web), pero este tema también lo trataremos más adelante. Ahora bien, es hora de discutir el rol del texto escrito como registro teatral.

\section{El texto escrito como registro teatral}

Para empezar, no podemos desconocer que el registro teatral que ha primado, ha sido la fotografía y/o el audiovisual, dando así origen a algunas de las morfologías más utilizadas de la performance, o sea el foto-performance y el vídeo-performance, respectivamente. Sin embargo, también podemos encontrar algunos casos en los que el texto escrito funciona como registro teatral de la pieza. Uno de ellos es El Préstamo (2000) de Aníbal López, pieza que fue presentada en una galería de la siguiente forma:
La obra no es más que un simple texto impreso sobre una manta de vinilo y que empieza de esta forma: "El día 29 de septiembre del 2000 realicé una acción que consistió en asaltar a una persona con apariencia de clase media". Sigue una descripción de la víctima, del modus operandi, y el monto robado, 874.35 quetzales. Termina asegurando que el vino que se están tomando los espectadores había sido comprado con ese dinero. (Escalón, 2014).

En este caso, al no existir ningún registro audiovisual (conocido), el texto escrito funciona como hábitat de la acción, pues este se despliega en la galería como un enunciado performativo en términos lingüísticos, esto es, que la emisión de dicha expresión implica la realización de una acción y no solo corresponde a meramente decir algo (Austin, 1962), lo que lo transforma así en el contenedor esencial de la pieza. Pero, pese a este ejemplo, el texto escrito como registro teatral no ha sido una práctica usual en la performance latinoamericana. Sin embargo, hay otro tipo de textos escritos que, si bien no cumplen directamente la función de registro, son relevantes para la actividad performática, cosa que pasaremos a revisar a continuación.

Paratextos escritos de la pieza de performance
A continuación, revisaremos aquellos textos escritos que circunscriben a la pieza performática, los que podemos denominar como paratextos. Pensemos que «etimológicamente, "paratexto" sería lo que rodea o acompaña al texto (para $=$ junto a, al lado de), aunque no sea evidente cuál es la frontera que separa texto de entorno» (Alvarado, 1994, p. 2).

Partamos por el título, parte esencial de cualquier obra de arte contemporánea. Si bien a veces este cumple solo un rol de identificación y clasificación de las piezas, en la mayoría de los casos desempeña una función semántica significativa. Por ejemplo, en piezas que recurren a un referente literario, el título puede ser clave para poder entregar dicha información, tal como sucede con 120 Piececitos de Niños Tomándose el Reino de Moda y Pueblo (2008), que refiere a dos textos de Gabriela Mistral, aunque al revisar la acción nos percatamos que se estructura prácticamente en su totalidad en torno a su poema Todas Íbamos a ser Reinas (Abdul, 2015).

Otro tipo de paratextos son los documentos escritos relacionados con el proceso de producción de las piezas, como los proyectos que los artistas redactan con el objetivo de acceder a financiamiento para ellas, los textos que ellos mismos escriben con el fin de empezar a tra- 
bajar en una obra determinada, facturas de compras necesarias para la pieza, afiches y panfletos que sirven para difundirla, entre otros. Esto nos obliga a entrar en el terreno de la literatura gris, que corresponde a "aquellos documentos que ofrecen una información informal, en formato no convencional" (Currás, 1998, p. 118), “documentos fugitivos, transparentes (que no se ven en los catálogos de editoriales, librerías, bibliotecas, etc.), de difícil localización que, sin embargo en la mayoría de los casos, contienen datos relevantes e importantes" (p. 119). De hecho, muchos de esos documentos nos permiten acceder a las piezas desde otros puntos de vista, entregando información sobre instancias creativas, y permitiendo analizar las formas de circulación y de gestión/autogestión de las mismas.

Otro tipo de paratexto son los textos que sirven de fundamento conceptual a las piezas, incluyendo los statements, los manifiestos, y también los textos curatoriales. El texto curatorial no tiene, como función primordial, dar cuenta de la obra o servir de hábitat de ella, sino que es un texto escrito que sirve de presentación y contextualización de una pieza artística. Por otra parte, están los textos escritos que acompañan a los registros audiovisuales disponibles en la Internet por medio de plataformas como YouTube, Vi- meo o páginas web, y que puntualizan o entregan información adicional sobre la pieza, situación que nos recuerda la fuerte vigencia del texto escrito pese a los avances tecnológicos.

\section{El texto escrito como espa-} cio de discusión de la performance

La historia y la teoría del arte se han articulado con base en lo escrito. Sin embargo, en el contexto latinoamericano pareciera ser que la producción de escritos críticos e históricos sobre performance no es ni de la calidad ni de la cantidad, respecto a la abundancia de piezas de performance. Además, muchos ensayos valiosos en el área no logran acceder a un grado de circulación significativo, pese a los esfuerzos de muchas publicaciones y de las instituciones que las apoyan. En esto influye la situación periférica de la performance en el marco de las artes visuales, aspecto que, sin embargo, la emparenta con la producción poética y que podría explicar el vínculo en la praxis entre ambas disciplinas (Arenas-Carter, 2018a). En efecto, Pueo (2012) afirma que:

El género lírico sea hoy el más minoritario de todos los géneros literarios -puede decirse que el número de lectores de poesía no es mucho mayor que el de los propios poetas, teniendo en cuenta que son éstos los consumido- res habituales de este tipo de literatura-, mantenido únicamente por el prestigio intelectual que se asocia a toda forma minoritaria de cultura. (pp. 439-440).

Por su parte, la performance ocupa una posición minoritaria en el campo de las artes visuales (Arenas-Carter, 2018a), lo que se puede explicar con base en factores como su relativamente corta historia, escasez de fondos estatales y privados para sustentar su producción, su incapacidad para ser mercantilizada directamente, y la falta de intermediación ante la audiencia. Sobre esto último, pensemos que la performance creativa se encuentra prácticamente ausente del currículum escolar de artes en prácticamente toda Latinoamérica, e incluso a nivel de posgrado, ya que contamos con pocos programas en el área de tipo maestría o doctorado. Además, y al igual que pasa con la literatura, se tiende a concentrar la producción crítica en unos pocos nombres, dejando de lado la posibilidad de explorar nuevos territorios.

Ahora bien, existen otros espacios de discusión de la performance como conferencias, seminarios y coloquios, a los que podemos acceder por medio de vídeos subidos en la Internet. Sin embargo, podemos pensar que el texto escrito seguirá teniendo un rol significativo a la hora de debatir sobre perfor- 
mance, conviviendo con nuevos espacios como los nombrados. Sin duda, seguiremos escribiendo para seguir pensando las piezas de performance, generando reflexiones sobre ellas no solo por medio del texto escrito, sino que también con base en información recogida a través de él.

Performance y texto escrito literario

Ahora bien, hay un aspecto en particular que debemos revisar debido a su conexión con la literatura. ¿Qué sucede cuando un texto escrito literario es el motor conceptual de una pieza? Como se señaló anteriormente, en Latinoamérica hay un grupo no menor de artistas visuales y literatos que producen tanto poesía como performance (Arenas-Carter, 2018a). Gran parte de ellos elaboran piezas de performance con base en textos de su autoría que funcionan de manera independiente a dichas piezas, como en el caso de Manule Tzoc. Su performance Erotika FM (2011) (M.Tzoc, entrevista personal, 1 de agosto de 2018) está basada e incluye textos de su poemario Gay-O (2010). Otro caso es el de Cecilia Vicuña, que en su pieza Vaso de Leche (1979), luego de la acción de escribir con tiza sobre el pavimento el poema homónimo, derrama un vaso con leche (Artishock, 2017).

Por otra parte, tenemos el recorrido profesional de artistas como Regina José Galindo y Eli Neira, quienes pese a que se han destacado más por su trabajo performático, no han dejado de publicar poemarios, mientras que otros que visitaron la performance creativa como Mario Bellatín, Pedro Lemebel y Raúl Zurita, optaron más por focalizarse en la literatura creativa. Al respecto, estos casos pueden incluso considerarse como inevitables, tal como lo afirma Tzoc (M.Tzoc, entrevista personal, 1 de agosto de 2018), en especial tomando en cuenta la relevancia de lo escritural en nuestra cultura, la vigencia en las prácticas de los artistas de performance de la ya comentada unión entre arte y vida que puede explicar ese devenir entre ambas disciplinas, las prácticas inscritas dentro de la literatura en el campo expandido, la ya señalada necesidad de reinvención del texto literario (Rodríguez Ruíz, 2018) y el lugar marginal que ocupan en la praxis la poesía y la performance dentro de sus correspondientes esferas (literatura y artes visuales) (Arenas-Carter, 2018a).

Por otro lado, hay creadores que elaboran sus propuestas performáticas con base en trabajos de otros autores, como es el caso de Diego Ramírez y su proyecto Moda y Pueblo, con talleres de poesía que incluyen la revisión teórica y práctica de la performance tanto como instrumento pedagógico, como para reinventar el texto literario, produciendo piezas con base en autores como la misma Vicuña (Ramírez, 2008) o Gabriela Mistral (Abdul, 2015), como sucede con la ya nombrada 120 Piececitos de Niños Tomándose el Reino.

En otros casos, la performance ha sido utilizada como recurso anexo a la presentación de libros, con resultados diversos y que a veces quedan en el limbo en el que habitan las piezas absolutamente subordinadas al texto al que aluden y/o al contexto de la actividad literaria en cuestión. Sin embargo, otras presentan una autonomía total, como el caso de la performance del colectivo argentino $L a$ Organización Negra en ZonaFuturo Feria del Libro 2016 , realizada a propósito de la presentación del ensayo escrito por Malala Rodríguez sobre el grupo (Performance de LON en ZonaFuturo Feria del Libro 2016), de la que incluso puede pensarse desempeñó un rol pedagógico para intentar comunicar el concepto de performance creativa a la audiencia presente.

Todos estos rasgos vuelven a denotar la estrecha relación entre producción textual literaria y producción de performance, en particular respecto a la poesía. Sobre esto, sería interesante explorar otros géneros tal como lo hiciera Diamela Eltit. En su performance Zona de Dolor (1980), ella lee extractos de su novela (entonces, en proceso) 
Lumpérica (1983) durante una noche estando dentro de un burdel (Hemispheric Institute), a la vez que establece un diálogo entre el motivo de la novela con el espacio de la performance.

Un punto importante sería especificar que la relación entre performance y texto escrito, $\mathrm{y}$ en particular respecto a la literatura, puede caracterizarse como intermedial. Al respecto, Rodríguez Ruíz (2018) plantea que la intermedialidad puede entenderse de tres formas. Primero, como transposición medial, o sea transformar un determinado producto mediático o su substrato en otro medio, como las adaptaciones de novelas en películas. Segundo, como hibridación de medios, es decir, prácticas tradicionales se mezclan entre sí y/o con medios asociados a las nuevas tecnologías, superando la mera contigüidad de dos o más manifestaciones materiales por medio de la integración. Tercero, como referencialidad a otros medios, por ejemplo, referir en un texto literario a una película o viceversa.

Si bien el caso de cada pieza de performance es diferente, para ilustrar este tema podemos revisar algunas de las ya nombradas. Por ejemplo, 120 Piececitos de Niños Tomándose el Reino corresponde a la primera, por ser una reinterpretación en la cual el reino Mistraliano deviene en la canonización literaria, representada por el espacio en el que se realiza la pieza (Biblioteca Nacional de Chile), en contrapunto con los poetas con coronas de cartón que publican sus textos en formatos efímeros como el fanzine y que a la vez leen poemas que tratan de la no-realización de los sueños de niñez y juventud (Abdul, 2015). Claramente, la literatura es el tema de esta pieza. En la segunda esfera de la intermedialidad, podemos ubicar a Jazz Palabra, en la cual la combinación de música, poesía y acción delinea esta proto-performance hasta el punto de fundir los elementos individuales en una sola unidad semántica. Sobre el tercer aspecto, en Erotika FM, Manuel Tzoc realiza la acción de vestirse frente a la audiencia tanto con prendas masculinas como femeninas, apelando no solo a la ambigüedad sexual plasmada en los textos de Gay-O, sino que también refiriendo al ánimo exhibicionista y voyerista de los poemas, sin caer en una mera reinterpretación del poemario en cuestión (M.Tzoc, entrevista personal, 1 de agosto de 2018).

Nuevas tecnologías, texto escrito y performance

Discutiremos brevemente en este apartado las posibilidades e implicancias que pueden tener las nuevas tecnologías para la relación en estudio. Más allá de la mera transposición medial planteada por Rodríguez Ruíz (2018) como el primer tipo de intermedialidad, sin duda se vislumbran nuevas perspectivas. Algunas son difíciles de discutir, dada la escasa exploración de ellas por parte de los creadores latinoamericanos, y si bien resulta tentador recurrir a ejemplificaciones foráneas, hemos decidido no hacerlo para respetar las directrices del presente trabajo.

Una de las implicancias relevantes puede derivar desde la idea de que "las herramientas serían prolongaciones, verdaderas extensiones del cuerpo" (Radrigán, 2015, p. 23). Si pensamos en los celulares, tablets, computadoras, entre otros, como herramientas, y a eso agregamos que en muchas de ellas el texto escrito desempeña un papel relevante, pensemos en lo que puede acontecer en el campo de la app-performance, o sea de piezas que utilizan las redes sociales para su realización. Por ejemplo, el caso de mi pieza Fracaso 2 (2019). Una semana antes de su realización convoqué, por medio de un posteo en mis redes sociales personales que combinaba un texto escrito explicativo con una imagen de mi persona, a todas las personas que me odiaban, que tenían algún resentimiento contra mí, o que no se atrevían a decirme

9 Para un extenso análisis de las conexiones entre la susodicha novela y la performance, ver Olea (2008). 
algo, a que me escribieran un mail con todo lo que quisieran decir(me) sobre mí (y que no se atrevían) de forma absolutamente anónima. Una semana después, leí a viva voz los mails que me llegaron, junto con otros documentos que certificaban tanto mis fracasos personales como profesionales, al mismo tiempo que la frase Yo Soy un Fracaso era tatuada en mi espalda (Arenas-Carter, 2019).

En este caso, el texto escrito tuvo un rol fundamental para contactar a los interesados, y dicho contacto fue gatillado en el espacio de las redes sociales. Si pensamos que yo estoy consciente de que mis redes sociales son prolongaciones de mi propio cuerpo, entraremos en un terreno cyborg cuyas implicancias dan para un estudio aparte. Lo que sí queda claro es que hay posibilidades para que la performance, las tecnologías y la escritura sean compañeros y no rivales, más aún considerando lo que hemos discutido anteriormente sobre la intermedialidad. También, el devenir del texto escrito en este marco nos lleva hacia el interesantísimo terreno del texto escrito alojado en la Internet, que no solo amplía las posibilidades para los creadores de performance, sino en especial para los creadores literarios (Rodríguez Ruíz, 2018). Son perspectivas que están en desarrollo, y cuyo futuro dependerá de las tecnologías que los crea- dores latinoamericanos decidan privilegiar.

\section{A modo de conclusión}

Queda claro que la relación entre texto escrito y performance, y entre literatura y artistas de performance, ha estado presente desde el origen de la actividad performática latinoamericana, y lo seguirá estando durante mucho tiempo más. Al respecto, puede ser interesante revisar en otros estudios dicha relación con base en territorios nacionales, e incluso llevarla a otros continentes y marcos culturales. Además, el uso del texto escrito como registro teatral se abre como un espacio poco explorado al que los creadores de performance deberían atender, además de todo el abanico de posibilidades que se abre tanto para ellos como para los escritores de literatura ante las nuevas tecnologías. Por otro lado, diversos creadores fluyen entre la literatura y la performance, estableciéndose un diálogo entre ellas que entrega posibilidades de exploración creativa, productiva y pedagógica para la literatura a partir de la performance, y viceversa. Dichas posibilidades son amplísimas. Basta considerar que, a excepción de Enrique Vila-Matas (Arenas-Carter, 2018a), se desconoce la existencia de autores de ficción en habla hispana que hayan utilizado alguna vez al artista de performance como personaje. Por todo esto, la aparente oposición entre texto escrito y performance queda bastante cuestionada, lo que además obliga a replantear la pedagogía de la performance en términos de incluir las posibilidades que texto escrito y texto literario pueden otorgar, a la manera que lo ha hecho el proyecto Moda y Pueblo.

Ahora bien, bajo la luz de la intermedialidad (en sus tres modalidades) se abren posibilidades para ambos campos creativos. Las necesidades de reinvención de la literatura pueden alimentarse del arte de la performance, incluyendo la video-performance y la app-performance, quedando abierta la invitación a generar proyectos a partir de esto. Es más, los creadores de literatura podrían recurrir a un intercambio que incluya conocer los procesos de creación performática, con el fin de ampliar las opciones de reinvención. Queda entonces pendiente la generación de espacios en los que se pueda facilitar este intercambio, como pueden ser encuentros, residencias de creación o seminarios interdisciplinares, entre otros.

De igual manera, queda pendiente el desafío de seguir escribiendo sobre performance desde y hacia Latinoamérica, para seguir contribuyendo a armar este corpus que está en constante construcción y que se puede alimentar a partir de documentos escritos y literarios pertinen- 
tes. En este sentido, las consideraciones expuestas pueden servir de base para continuar y ampliar el debate, en especial al adoptar el autor un punto fluido entre la creación y la teoría (Borrero, 2012).

\section{Referencias}

Abdul, L. [Lucien Abdul]. (2015). 120 piececitos de niños tomándose el reino [Archivo de vídeo]. Recuperado de http://www.dailymotion.com/video/x2qub92

Artishock. (2017). Círculo ciclo/Cecilia Vicuña. Recuperado de http://artishockrevista.com/2017/08/28/ cecilia-vicuna-campus-creativo/

Alvarado, M. (1994). Paratexto. Buenos Aires: Instituto de Lingüística, U.B.A.

Arenas, R. (2014). New York City by the perspective of its new inhabitants: a descriptive study of the urban environment during the last fifty years into the U.S. Latino/a narrative. (Tesis de Maestría en Literatura). UPLA, Chile, Valparaíso.

Arenas-Carter, R. (2017). Olvido. Recuperado de https://rodsands.weebly.com/olvido.html

Arenas-Carter, R. (2018a). La vital precariedad. Poesía y performance en América Latina y Chile. Berlín: Editorial Académica Española.

Arenas-Carter, R. (2018b). Todo depende del aparato con que se mire: reflexiones en torno a performance, registro y tiempo. Manuscrito presentado para su publicación.

Arenas-Carter, R. (2018c). Diario (Terapia). Recuperado de https://terapiaperformance.weebly.com/ diario

Arenas-Carter, R. (2019). Fracaso 2. Recuperado de https://rodsands.weebly.com/fracaso2.html

Alberto Greco Biografía. (s.f.). Recuperado de http://www.albertogreco.com/es/bio/albertogreco.pdf

Alberto Greco ;Que grande sos! (s.f.). Recuperado de http://www.albertogreco.com/es/obras/artevivo/ quegrandesos/index.htm

Arroyave, M. M., y Barreto Mesa, M. (2005). De las estrategias de aprendizaje a la composición de textos expositivos/descriptivos: una propuesta de intervención pedagógica. (Tesis de Maestría). Universidad de Antioquia, Medellín, Colombia.

Auslander, P. (1999). Liveness. Performance in a mediatized culture. Oxon: Routledge. 
Auslander, P. (2006). The performativity of performance documentation. PAJ: A Journal of Performance and Art, 28(3), 1-10.

Austin, J. L. (1962). How to do things with words. Londres: Oxford University Press.

Borrero, J. (2012). «Muchacha no vayas al bosque»: orientaciones para una literatura en el campo expandido. La Palabra, (20), 49-67. Recuperado de https://revistas.uptc.edu.co/index.php/la_palabra/ article/view/958

Brijaldo Olarte, G. (2014). Interpretaciones íntimas sobre la escritura performativa. La Palabra, (24), 111-117. Doi: https://doi.org/10.19053/01218530.2506

Carrillo, C. V. (2007). Grupos artístico-literarios en la Venezuela de los años sesenta. Latinoamérica. Revista de estudios latinoamericanos, (44), 59-81.

Cullen, D. (2011). Arte $\neq$ vida a chronology of actions by artists of the Americas, 1960-2000. E-misférica, Hemispheric Institute of Performance and Politics. Recuperado de http://archive.hemisphericinstitute. org/web-cuadernos/arte-no-es-vida

Currás, E. (1998). Sistema experto hipermedia para el tratamiento de literatura gris. Scire: representación y organización del conocimiento, 4(1), 117-130.

Escritos: Besos Brujos. (s.f.). Recuperado de http://www.albertogreco.com/es/obras/escritos/ besosbrujos/index.htm

Escalón, S. (2014). El artista que contrató a un sicario. Ciudad de Guatemala: Plaza Pública. Recuperado de http://www.plazapublica.com.gt/content/el-artista-que-contrato-un-sicario

García, A. (2007). El efimero pánico. Recuperado de http://artesescenicas.uclm.es/index. php?sec $=$ texto $\& \mathrm{id}=66$

Halliday, M. (1994). Spoken and written modes of meaning. Media texts: Authors and readers, 7, 51-73.

Hemispheric Institute. (s.f.). Diamela Eltit: Zona de dolor. Recuperado de http://hemisphericinstitute. org/hemi/es/hidvl-additional-performances/diamela-eltit-zona-de-dolor

Jones, A. (1997). "Presence" in absentia: experiencing performance as documentation. Art Journal, 56(4), 11-18.

Leanfilms. (2009). Arte Calle Group. Most Famous Performance [Archivo de vídeo]. Recuperado de https://www.youtube.com/watch?v=LFO_RDnv3z4 
López-Cabrales, M. (2006). Laberintos corporales en la obra de Ana Mendieta. Espéculo, (33). Recuperado de http://webs.ucm.es/info/especulo/numero33/laberint.html

Martos Núñez, E., \& Vivas, A. (Coords.) (2010). Performance, lectura y escritura (Conceptos y prácticas). Madrid: Universidad Complutense, Red Internacional de Universidades Lectoras, Colección Temas de Lectura.

Manifiesto Dito del Arte Vivo (s.f.). Recuperado de http:/www.albertogreco.com/es/obras/artevivo/ manifiestodito/index.htm

Marilyn Boror-art. (2018, 27 de septiembre). Edicto cambio de nombre. Recuperado de https://www. facebook.com/artemarilynboror/photos/a.1482411018681598/2147849408804419/?type=3\&th eater

Nesseldore, S. (2014). Defining the Aesthetic (s) of Negation in El Techo de la Ballena. Caiana. Revista de Historia del Arte y Cultura Visual del Centro Argentino de Investigadores de Arte (CAIA), (4), 1-8.

Olea, R. (2008). Contrapuntos narrativos. Lenguaje verbal e imagen visual en Lumpérica de Diamela Eltit. Taller de Letras, (43), 175-187.

Pereztejada, J.J. (2014). Jazz Palabra, un espectáculo de Juan José Gurrola. Recuperado de https:// esquesoydeveracruz.blogspot.com/2014/04/jazz-palabra-un-espectaculo-de-juan.html

Performance de LON en ZonaFuturo Feria del Libro 2016. (s.f.). Recuperado de https://interzonaeditora. com/noticias/performance-de-lon-en-zonafuturo-feria-del-libro-2016-405

Pueo, J. (2012). Más allá de la Lectura. Reseña de "Performing Poetry: Body, Place and Rhythm in the Poetry Performance". Tropelías: Revista de Teoría de la Literatura y Literatura Comparada, (18), 439-442.

Radrigán, V. (2015). Tecnomorfosis: desbordes e hibridaciones entre el cuerpo y la tecnología. (Tesis de Doctorado). Universidad de Chile, Santiago, Chile.

Reisz de Rivarola, S. (1981). Texto literario, texto poético, texto lírico: elementos para una tipología. Lexis, 5(2), 1-34.

Rama, Á. (1998). La ciudad letrada. Montevideo: Arca.

Ramírez, D. (2008). ¿Quieres hacerme ver el cielo? Recuperado de http://moda-pueblo.blogspot. com/2008/01/quieres-hacerme-ver-el-cielo.html

Ramírez, J. (2018). Regina José Galindo o el cuerpo como nación. Boletín de arte, (30-31), 519-532. 
Ríos, M. C. (2018). La escritura dinámica de Mario Bellatin: del texto al teatro y del teatro al texto. $L a$ Palabra, (32), 99-116. Doi: https://doi.org/10.19053/01218530.n32.2018.8168

Rodríguez, M. (2009). María Teresa Hincapié y el "Actor Santo": sacralizar lo cotidiano. Antípoda. Revista de Antropología y Arqueología, (9), 113-130.

Rodríguez Ruíz, J. A. (2018). Del autor junta palabras al autor junta palabras, imágenes, sonidos, interactividades, algoritmos: la gestión literaria en la era (post) digital. La Palabra, (32), 19-41. Doi: https://doi.org/10.19053/01218530.n32.2018.8159

Taylor, D. (2015). El archivo y repertorio. La memoria cultural performática en las Américas. Santiago de Chile: Ediciones Universidad Alberto Hurtado.

Wilson, M. (1997). Performance art: (some) theory and (selected) practice at the end of this century. Art Journal, 56(4), 2-3. 